\title{
Ability of 1,5-Anhydro-d-glucitol Values to Predict Coronary Artery Disease in a Non-Diabetic Population
}

\author{
Nobutaka IKedA, ${ }^{1} \mathrm{MD}$, Hisao Hara, ${ }^{1} \mathrm{MD}$, and Yukio HiroI, ${ }^{1} \mathrm{MD}$
}

\begin{abstract}
SUMmary
Increasing evidence has indicated that postprandial hyperglycemia affects coronary artery disease (CAD). The serum 1,5-anhydro-d-glucitol (1,5-AG) value is a useful clinical marker to evaluate short-term glycemic status and reflects glycemic excursions with greater sensitivity when compared with hemoglobinAlc (HbAlc), especially for patients in the postprandial state. The aim of this study was to evaluate the predictive value of 1,5-AG for CAD in patients without diabetes mellitus.

This study included 729 consecutive patients who had undergone their first coronary angiography. A total of 284 patients (246 diabetic patients and 38 patients with stage 4 or 5 chronic kidney disease) were excluded. The predictive values of 1,5-AG and $\mathrm{HbA1c}$ for $\mathrm{CAD}$ were evaluated by multivariable logistic regression analysis.

Patients with $\mathrm{CAD}$ demonstrated significantly lower 1,5-AG values and higher HbA1c values than did patients without CAD $(18.6 \mu \mathrm{g} / \mathrm{mL}[12.0,23.3]$ versus $19.2 \mu \mathrm{g} / \mathrm{mL}[14.4,25.2], P=0.036$, and 5.7\% [5.5, 5.9] versus 5.6\% [5.4, 5.8], $P=0.016$, respectively). In multivariable logistic regression analysis, the $\mathrm{HbAlc}$ values did not indicate a predictive value for the prevalence of $\mathrm{CAD}$. In contrast, the 1,5-AG levels were still an independent predictor of CAD (adjusted odds ratio $0.96,95 \%$ confidence interval $0.93-0.99, P=0.0097$ ).
\end{abstract}

Serum 1,5-AG is superior to $\mathrm{HbAlc}$ for predicting CAD prevalence in patients without diabetes mellitus. (Int Heart J 2015; 56: 587-591)

Key words: Glucose, Ischemic heart disease

$\mathrm{T}$ ype 2 diabetes mellitus (DM) worsens the morbidity and mortality of patients with cardiovascular disease (CVD). ${ }^{1}$ Because of the established association between glycated hemoglobin (HbAlc) and microvascular disease, the American Diabetes Association recommends the evaluation of $\mathrm{HbAlc}$ as a criterion for diagnosing DM. ${ }^{2)}$ $\mathrm{HbA1c}$ values can be evaluated in a non-fasting state, are highly reproducible, and are considered to be useful markers of long-term glycemic control. Furthermore, elevated HbA1c values are associated with increased prevalence and complexity of coronary artery disease (CAD), polyvascular disease, future microvascular and macro-vascular complications, and mortality ${ }^{3-6)}$ However, in patients with established DM, intensive intervention for glycemic control guided by $\mathrm{HbAlc}$ values did not improve the risk of macro-vascular complications and survival prognosis. ${ }^{7-9)}$ To obtain favorable effects for protection from macro-vascular complications, early diagnosis and early intervention to correct glycemic abnormalities are essential. ${ }^{10}$ Increasing evidence has indicated that postprandial hyperglycemia affects CVD progression. ${ }^{11,12)}$ The DECODE trial demonstrated the association between postprandial hyperglycemia and mortality with no relation to fasting glucose levels. ${ }^{13)}$

The serum 1,5-anhydro-d-glucitol (1,5-AG) level rapidly decreases concomitantly with urinary glucose excretion and is an important and feasible clinical marker of short-term glycemic status. ${ }^{14,15)}$ A previous report showed that $1,5-\mathrm{AG}$ is superior to $\mathrm{HbAlc}$ as a predictor of $\mathrm{CAD}$ prevalence. ${ }^{16)}$ However, no report has examined the predictive value of 1,5-AG for $\mathrm{CAD}$ in a non-diabetic population. The aim of this study was to evaluate the predictive values of serum 1,5-AG levels for $\mathrm{CAD}$ prevalence in non-diabetic adults.

\section{MeTHODS}

Study patients: This study included 729 consecutive patients who were admitted to the National Center for Global Health and Medicine between July 2011 and August 2014 and had undergone their first coronary angiography. Coronary angiography was performed to assess ischemic heart disease, cardiomyopathy, arrhythmia, and congestive heart failure and to preoperatively investigate for ischemic heart and aortic or valvular disease. A total of 445 patients were included for analysis after excluding 246 diabetic patients and 38 patients with stage 4 or 5 chronic kidney disease. Diabetes mellitus was defined as fasting plasma glucose $\geq 126 \mathrm{mg} / \mathrm{dL}$ and $\mathrm{HbAlc} \geq 6.5 \% \mathrm{Na}$ tional Glycohemoglobin Standardization Program (NGSP), or the use of an oral hypoglycemic agent or insulin. This study

From the ${ }^{1}$ Division of Cardiovascular Medicine, National Center for Global Health and Medicine, Tokyo, Japan

Address for correspondence: Nobutaka Ikeda, MD, Division of Cardiovascular Medicine, National Center for Global Health and Medicine, 1-21-1 Toyama, Shinjukuku, Tokyo 162-8655, Japan. E-mail: ikedanobutaka@yahoo.co.jp

Received for publication April 30, 2015. Revised and accepted May 9, 2015.

Released in advance online on J-STAGE November 6, 2015.

All rights reserved by the International Heart Journal Association. 
Table I. Patient Characteristics

\begin{tabular}{lcccc}
\hline \multicolumn{1}{c}{ Variable } & All patients $n=445$ & CAD(-) $n=283$ & CAD(+) $n=162$ & $P$ \\
\hline Male sex (\%) & 63 & 58 & 72 & 0.0049 \\
Age & $71(62,79)$ & $69(60,78)$ & $75(66,80)$ & 0.0005 \\
Hypertension (\%) & 59 & 52 & 72 & $<0.0001$ \\
Dyslipidemia (\%) & 49 & 44 & 58 & 0.0086 \\
Current smoking (\%) & 49 & 49 & 50 & 0.77 \\
Total cholesterol (mg/dL) & $181(158,207)$ & $178(153,201)$ & $186(164,209)$ & 0.025 \\
LDL cholesterol (mg/dL) & $108(88,126)$ & $104(85,125)$ & $112(92,130)$ & 0.0089 \\
HDL cholesterol (mg/dL) & $50(40,61)$ & $51(41,62)$ & $48(39,57)$ & 0.020 \\
Triglycerides (mg/dL) & $100(71,140)$ & $99(69,142)$ & $101(77,149)$ & 0.20 \\
Serum glucose (mg/dL) & $101(92,114)$ & $100(91,112)$ & $103(92,118)$ & 0.093 \\
eGFR (mL/minute/1.73 m $\left.{ }^{2}\right)$ & $67(57,78)$ & $68(58,78)$ & $64(53,77)$ & 0.027 \\
\hline
\end{tabular}

LDL indicates low density lipoprotein; HDL, high density lipoprotein; and eGFR, estimated glomerular filtration ratio.

complied with the Declaration of Helsinki, and written informed consent was obtained from each patient. The local ethics committee approved the use of clinical data for this study. 1,5-AG and HbA1c measurements: The 1,5-AG levels were measured by colorimetric analysis using a Lana 1,5-AG auto liquid automatic analyzer (JCA-BM8060, JEOL Ltd.).

$\mathrm{HbA1c}$ levels were measured by high-performance liquid chromatography using an HA-8180 (ARKRAY). From July 2011 to March 2012, HbA1c levels were calculated with the NGSP equivalent levels using the following formula: HbA1c $(\operatorname{NGSP})(\%)=1.02 \times$ HbA1c (Japan Diabetes Society) $(\%)+0.25 \% .{ }^{17)}$ NGSP levels were directly obtained from April 2012 to August 2014.

Angiographic analysis: Coronary arteriography was performed by experienced cardiologists using standard techniques in all study patients. All of the coronary arteries were injected, and at least 2 views of the right arteries and 4 views of the left arteries were collected. The prevalence of CAD was assessed by two or three experienced interventional cardiologists who were blinded to the clinical data. The CAD prevalence was defined as the existence of significant stenosis (more than $75 \%$ stenosis by visual estimation) in main epicardial coronary arteries. When the CAD assessment showed a discrepancy between 2 observers, the opinion of a third observer was required, and the final decision was made by consensus of all 3 observers.

Coronary lesion complexity was assessed by the SYNTAX score. According to the diagnostic coronary angiogram, each coronary artery lesion with more than 50\% diameter stenosis in vessels $1.5 \mathrm{~mm}$ and longer was separately scored, and these scores were summed to obtain the overall SYNTAX score, which was calculated using the SYNTAX score algorithm. The SYNTAX score algorithm is available on the SYNTAX website. ${ }^{18)}$ The SYNTAX scores obtained were evaluated by 2 or 3 experienced interventional cardiologists who were blinded to the clinical data. In the event of a disagreement, the opinion of a third observer was required, and the final decision was made by consensus.

Statistical analysis: The Kolmogorov-Smirnov test was used to evaluate normal distribution. The continuous variables are presented as the median and interquartile range (median [25th, 75 th percentiles]). The categorical variables are presented as counts or proportion (percentage). The Mann-Whitney $U$ test was used to compare the continuous variables. To evaluate the predictive values for $\mathrm{CAD}$ prevalence, logistic regression anal- ysis (forced entry method) was used. In multivariate analysis, sex, age, hypertension, dyslipidemia, current smoking status, $\mathrm{HbA1c}$ level, and 1,5-AG level were adjusted. These variables were considered traditional coronary risk factors and main concerns in this study. Correlations between the SYNTAX scores and 1,5-AG values were analyzed by Spearman's rank correlation coefficient. A $P$-value $<0.05$ was considered to be significant. SPSS ver. 22 software (IBM Japan, Tokyo) was used for the analyses.

\section{RESULTS}

Patient characteristics: The median patient age $(n=445)$ was 71 years, $280(63 \%)$ patients were male, and 162 patients (36\%) had CAD. The patients with CAD had a significantly higher age and a higher prevalence of male sex, hypertension, and dyslipidemia (Table I).

The median values of 1,5-AG and $\mathrm{HbA} 1 \mathrm{c}$ of all study patients were $19.1 \mu \mathrm{g} / \mathrm{mL}[13.5,24.2]$ and $5.6 \%$ [5.4, 5.9], respectively (median [25th, 75th percentiles]). Patients with CAD demonstrated significantly lower 1,5-AG values and higher HbA1c values than patients without CAD $(18.6 \mu \mathrm{g} / \mathrm{mL}$ [12.0, 23.3] versus $19.2 \mu \mathrm{g} / \mathrm{mL}[14.4,25.2], P=0.036$, and $5.7 \%[5.5,5.9]$ versus $5.6 \%[5.4,5.8], P=0.016$, respectively) (Figure).

Predictive values of 1,5-AG and HbA1c measurements for the prevalence of coronary artery disease: According to univariate logistic regression analysis, male sex, age, hypertension, dyslipidemia, and 1,5-AG and $\mathrm{HbA} 1 \mathrm{c}$ levels were predictors of $\mathrm{CAD}$ prevalence. However, after adjusting for all of these variables, the HbA1c levels did not have a predictive value for the prevalence of CAD. In contrast, the 1,5-AG levels were still an independent predictor of CAD (multivariable adjusted odds ratio $0.96,95 \%$ confidence interval $0.93-0.99, P=0.0097$ ) (Table II).

The median value [interquartile range] of the SYNTAX scores in patients with CAD was $11[6,22]$. There was no correlation between 1,5-AG or HbA1c values and the SYNTAX scores in patients with $\operatorname{CAD}(\rho=0.10, P=0.20$ and $\rho=0.12$, $P=0.15$, respectively). 

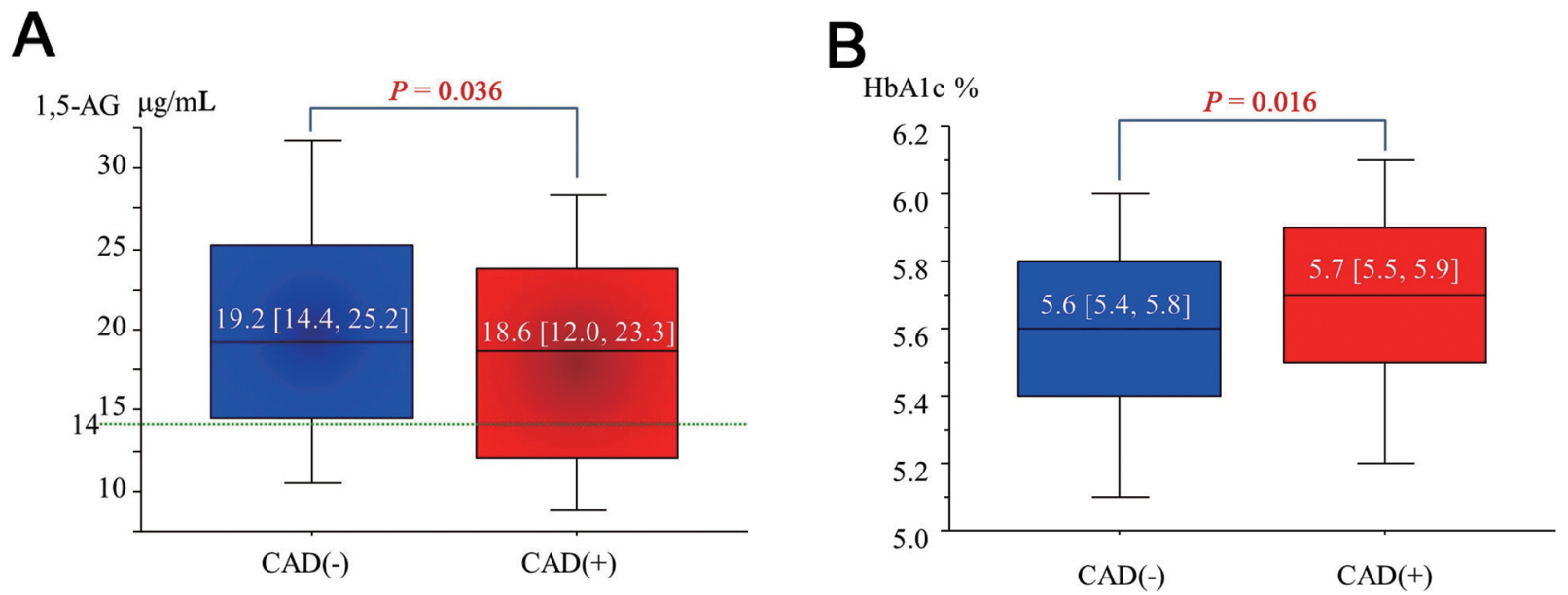

Figure. A: 1,5-AG values of patients with/without coronary artery disease (CAD). The widely accepted cut-off value for the detection of glycometabolic disorder $(14 \mu \mathrm{g} / \mathrm{mL})$ is within the 25 th percentile of 1,5-AG values of the patients with and without CAD. B: HbAlc values of patients with/without CAD.

Table II. Predictors of Patients With Coronary Artery Disease

\begin{tabular}{lcccccccc}
\hline & $\begin{array}{c}\text { Unadjusted odds } \\
\text { ratio (95\%CI) }\end{array}$ & $P$ & $\begin{array}{c}\text { Adjusted odds } \\
\text { ratio (95\%CI) } \\
\text { Model 1 }\end{array}$ & $P$ & $\begin{array}{c}\text { Adjusted odds } \\
\text { ratio (95\%CI) } \\
\text { Model 2 }\end{array}$ & $P$ & $\begin{array}{c}\text { Adjusted odds } \\
\text { ratio (95\%CI) } \\
\text { Model 3 }\end{array}$ & $\begin{array}{c}P \\
P\end{array}$ \\
\hline Male sex & $1.81(1.19-2.74)$ & 0.0051 & $3.01(1.79-5.06)$ & $<0.0001$ & $2.39(1.44-3.98)$ & 0.0008 & $2.68(1.58-4.53)$ & 0.0002 \\
Age & $1.03(1.02-1.05)$ & 0.0002 & $1.04(1.02-1.06)$ & 0.0001 & $1.04(1.02-1.06)$ & $<0.0001$ & $1.04(1.01-1.06)$ & 0.0008 \\
Hypertension & $2.34(1.55-3.53)$ & $<0.0001$ & $1.68(1.07-2.64)$ & 0.026 & $1.82(1.14-2.89)$ & 0.012 & $1.77(1.11-2.82)$ & 0.017 \\
Dyslipidemia & $1.69(1.14-2.50)$ & 0.0088 & $1.95(1.26-3.00)$ & 0.0027 & $1.83(1.17-2.85)$ & 0.0076 & $1.79(1.14-2.81)$ & 0.011 \\
Current smoking & $1.06(0.71-1.57)$ & 0.77 & $0.93(0.59-1.48)$ & 0.77 & $0.91(0.57-1.45)$ & 0.69 & $0.97(0.60-1.56)$ & 0.89 \\
HbA1c & $1.87(1.09-3.19)$ & 0.023 & & & $1.30(0.73-2.30)$ & 0.37 & $1.45(0.79-2.68)$ & 0.23 \\
1,5-AG & $0.97(0.95-0.99)$ & 0.012 & $0.96(0.94-0.99)$ & 0.013 & & & $0.96(0.93-0.99)$ & 0.0097 \\
\hline
\end{tabular}

Model 1: Male sex, age, hypertension, dyslipidemia, current smoking, and 1,5-AG were adjusted. Model 2: Male sex, age, hypertension, dyslipidemia, current smoking, and HbA1c were adjusted. Model 3: Model 2 plus 1,5-AG were adjusted.

\section{Discussion}

The principal finding of this study is that the predictive value of 1,5-AG levels for CAD prevalence was superior to the predictive value of $\mathrm{HbA} 1 \mathrm{c}$ levels in non-diabetic patients. In a previous report, we demonstrated the superiority of 1,5-AG over $\mathrm{HbA} 1 \mathrm{c}$ for predicting $\mathrm{CAD}$ among elective coronary angiography candidates. ${ }^{16)}$ However, no previous study has demonstrated the predictive value of 1,5-AG for CAD in a non-diabetic population. Watanabe, et al reported the association between 1,5-AG values and high incidences of strokes, ischemic strokes, and all cardiovascular diseases in non-diabetic men. However, no correlation was found between 1,5-AG values and the incidences of coronary heart diseases. ${ }^{19)}$

Undiagnosed DM or postprandial hyperglycemia has a progressive impact on vascular complications. ${ }^{4,20-23)}$ Microvascular DM complications are strongly associated with HbA1c levels. ${ }^{3,4)}$ In contrast, macrovascular complications develop in the early stages of glycometabolic disorders and do not linearly correlate with $\mathrm{HbA1c}$ values. ${ }^{6,24}$

In the 1990s, intensive glucose lowering treatments for patients with DM demonstrated protective effects on microvascular complications but did not decrease macro-vascular complications (the Diabetes Control and Complications Trial; DCCT, Kumamoto study, UK Prospective Diabetes Study;
UKPDS33). ${ }^{25-27)}$ Long-term follow-up of these patients was needed to obtain favorable effects on macro-vascular complications from intensive glucose lowering treatment, the socalled "Legacy effect". ${ }^{10,28)}$ However, in patients with highly established DM, HbAlc level-guided intensive interventions for glucose control did not reduce the risk of macro-vascular complications and even diminished survival prognosis. ${ }^{7-9)}$ However, in the DECODE trial, hyperglycemia in oral glucose tolerance tests was associated with mortality and had no relationship with fasting glucose levels. ${ }^{13)}$ Intervention with the alpha-glucosidase inhibitor acarbose reduced the risk of myocardial infarction and cardiovascular disease in patients with type $2 \mathrm{DM}$ while most patients were already receiving intensive concomitant cardiovascular medication. ${ }^{29)}$ Therefore, early diagnosis and early intervention to postprandial hyperglycemia may improve the prognosis of patients with cardiovascular disease.

The results of this study suggest that ischemic coronary artery disease progresses in the early stage of glycometabolic disorder, which could not be detected by HbA1c values. Furthermore, the 1,5-AG value can detect abnormal glucose metabolism to which HbA1c levels do not respond. In this study, the median values of 1,5-AG in patients with CAD and without CAD were $18.6 \mu \mathrm{g} / \mathrm{mL}$ and $19.2 \mu \mathrm{g} / \mathrm{mL}$, respectively. There was only a slight difference between the two groups be- 
cause the study population was limited to non-DM patients. However, the widely accepted 1,5-AG cut-off value of $14.0 \mu \mathrm{g} /$ $\mathrm{mL}$ is within the 25 th percentile of $1,5-\mathrm{AG}$ values of the patients with and without CAD (Figure A). Therefore, when 1,5AG values are used during routine health checkups, its usefulness will be further emphasized. Thus, the $\mathrm{HbAlc}$ value is a marker for the quantity of glucose control and 1,5-AG is a marker for the quality of glucose control. 1,5-AG values can be obtained from a non-fasting blood sample and therefore may be used as a useful and feasible alternative to continuous glucose monitoring and oral glucose tolerance tests.

In contrast, 1,5-AG values may not be suitable to predict the complexity of CAD in non-DM patients. In our results, there was no significant correlation between 1,5-AG values and the SYNTAX scores in patients with CAD. In this study, the median value and interquartile range of the SYNTAX scores for CAD patients was $11 .^{6,22)}$ This result indicates that most of the CAD patients in our study had so-called "Low SYNTAX lesions". In addition, because our study patients were non-DM, variation among the 1,5-AG values was also small. Therefore, both 1,5-AG values and the SYNTAX scores had little variation, and it was difficult to demonstrate a correlation between the two measurements.

Study limitations: There were some limitations in this study. First, because the study population included candidates for first-time coronary angiography, these results may have been subject to selection bias. Therefore, our results may not be directly applicable to the general population or to patients with other cardiovascular diseases. Second, $99.8 \%$ of the study patients were Asian. Third, there was an interaction between the 1,5-AG value and eGFR value. Therefore, eGFR was not included in variables of logistic regression analysis (Table II).

Clinical implications: Cardiologists should be aware of the risks of glycometabolic derangement in patients without established diabetes mellitus. Early diagnosis and interventions for glucose control are essential to reduce CVD risks. ${ }^{10)}$ The 1,5AG measurement is superior to HbAlc values for the detection of coronary artery disease, even in patients without diabetes mellitus. 1,5-AG values measured in mass will provide favorable additional values for the early diagnosis of coronary artery disease in the non-diabetic population.

Conclusion: Serum 1,5-AG values are superior to $\mathrm{HbA} 1 \mathrm{c}$ values for predicting CAD prevalence in patients without diabetes mellitus.

\section{Disclosure}

Conflicts of interest: None

\section{REFERENCES}

1. Kannel WB, McGee DL. Diabetes and cardiovascular disease. The Framingham study. JAMA 1979; 241: 2035-8.

2. American Diabetes Association. Diagnosis and classification of diabetes mellitus. Diabetes Care 2010; 33: S62-9.

3. Irie Y, Sakamoto K, Kubo F, et al. Association of coronary artery stenosis with carotid atherosclerosis in asymptomatic type 2 diabetic patients. J Atheroscler Thromb 2011; 18: 337-44.

4. Stolar M. Glycemic control and complications in type 2 diabetes mellitus. Am J Med 2010; 123: S3-11. (Review)
5. Selvin E, Steffes MW, Zhu H, et al. Glycated hemoglobin, diabetes, and cardiovascular risk in nondiabetic adults. N Engl J Med 2010; 362: 800-11

6. Ikeda N, Iijima R, Hara H, Moroi M, Nakamura M, Sugi K. Glycated hemoglobin is associated with the complexity of coronary artery disease, even in non-diabetic adults. J Atheroscler Thromb 2012; 19: 1066-72.

7. Patel A, MacMahon S, Chalmers J, et al. Intensive blood glucose control and vascular outcomes in patients with type 2 diabetes. $\mathrm{N}$ Engl J Med 2008; 358: 2560-72.

8. Duckworth W, Abraira C, Moritz T, et al. Glucose control and vascular complications in veterans with type 2 diabetes. $\mathrm{N}$ Engl $\mathrm{J}$ Med 2009; 360: 129-39.

9. Gerstein HC, Miller ME, Byington RP, et al. Effects of intensive glucose lowering in type 2 diabetes. N Engl J Med 2008; 358 : 2545-59.

10. Holman RR, Paul SK, Bethel MA, Matthews DR, Neil HA. 10year follow-up of intensive glucose control in type 2 diabetes. $\mathrm{N}$ Engl J Med 2008; 359: 1577-89.

11. Bonora E, Muggeo M. Postprandial blood glucose as a risk factor for cardiovascular disease in Type II diabetes: the epidemiological evidence. Diabetologia 2001; 44: 2107-14. (Review)

12. Ceriello A. The possible role of postprandial hyperglycaemia in the pathogenesis of diabetic complications. Diabetologia 2003; 4: M9-16. (Review)

13. Glucose tolerance and mortality: comparison of $\mathrm{WHO}$ and American Diabetes Association diagnostic criteria. The DECODE study group. European Diabetes Epidemiology Group. Diabetes Epidemiology: Collaborative analysis Of Diagnostic criteria in Europe. Lancet 1999; 354: 617-21.

14. Yamanouchi T, Akanuma Y. Serum 1,5-anhydroglucitol (1,5 AG): new clinical marker for glycemic control. Diabetes Res Clin Pract 1994; 24: S261-8. (Review)

15. Dungan KM. 1,5-anhydroglucitol (GlycoMark) as a marker of short-term glycemic control and glycemic excursions. Expert Rev Mol Diagn 2008; 8: 9-19. (Review)

16. Ikeda N, Hara H, Hiroi Y. 1,5-Anhydro-d-glucitol predicts coronary artery disease prevalence and complexity. J Cardiol 2014; 64: 297-301.

17. Kashiwagi A, Kasuga M, Araki E, et al. International clinical harmonization of glycated hemoglobin in Japan: From Japan Diabetes Society to National Glycohemoglobin Standardization Program values. J Diabetes Invest 2012; 3: 39-40.

18. Sianos G, Morel MA, Kappetein AP, et al. The SYNTAX Score: an angiographic tool grading the complexity of coronary artery disease. EuroIntervention 2005; 1: 219-27.

19. Watanabe M, Kokubo Y, Higashiyama A, Ono Y, Miyamoto Y, Okamura T. Serum 1,5-anhydro-D-glucitol levels predict first-ever cardiovascular disease: an 11-year population-based cohort study in Japan, the Suita study. Atherosclerosis 2011; 216: 477-83.

20. Tapp RJ, Shaw JE, Zimmet PZ, et al. Albuminuria is evident in the early stages of diabetes onset: results from the Australian Diabetes, Obesity, and Lifestyle Study (AusDiab). Am J Kidney Dis 2004; 44: 792-8.

21. Donahue RP, Abbott RD, Reed DM, Yano K. Postchallenge glucose concentration and coronary heart disease in men of Japanese ancestry: Honolulu Heart Program. Diabetes 1987; 36: 689-92.

22. Rodriguez BL, Lau N, Burchfiel CM, et al. Glucose intolerance and 23-year risk of coronary heart disease and total mortality: the Honolulu Heart Program. Diabetes Care 1999; 22: 1262-5.

23. Kato T, Node K. Therapeutic potential of $\alpha$-glucosidase inhibitors to prevent postprandial endothelial dysfunction. Int Heart J 2014; 55: 386-90. (Review)

24. Chou CH, Tsai WC, Wang MC, et al. Effects of deranged glucose homeostasis on peripheral arterial stiffness index in patients with pre-diabetes mellitus. Int Heart J 2013; 54: 27-32.

25. The effect of intensive treatment of diabetes on the development and progression of long-term complications in insulin-dependent diabetes mellitus. The Diabetes Control and Complications Trial Research Group. N Engl J Med 1993; 329: 977-86. 
26. Ohkubo Y, Kishikawa H, Araki E, et al. Intensive insulin therapy prevents the progression of diabetic microvascular complications in Japanese patients with non-insulin-dependent diabetes mellitus: a randomized prospective 6-year study. Diabetes Res Clin Pract 1995; 28: 103-17.

27. Intensive blood-glucose control with sulphonylureas or insulin compared with conventional treatment and risk of complications in patients with type 2 diabetes (UKPDS 33). UK Prospective Dia- betes Study (UKPDS) Group. Lancet 1998; 352: 837-53.

28. Nathan DM, Cleary PA, Backlund JY, et al. Intensive diabetes treatment and cardiovascular disease in patients with type 1 diabetes. N Engl J Med 2005; 353: 2643-53.

29. Hanefeld M, Cagatay M, Petrowitsch T, Neuser D, Petzinna D, Rupp M. Acarbose reduces the risk for myocardial infarction in type 2 diabetic patients: meta-analysis of seven long-term studies. Eur Heart J 2004; 25: 10-6. 